\title{
Power and Sample Size Determination for a Stepwise Test Procedure for Finding the Maximum Safe Dose
}

\author{
Ajit C. Tamhane ${ }^{1}$ \\ Department of IE/MS and Department of Statistics \\ Northwestern University \\ Evanston, IL 60208 \\ USA
}

Kunyang Shi

Department of Statistics

Northwestern University

Evanston, IL 60208

USA
Klaus Strassburger

Department of Biometrics \& Epidemiology German Diabetes Research Institute

Duesseldorf University

GERMANY

August 27, 2003

\footnotetext{
${ }^{1}$ Corresponding Author. E-mail address: ajit@iems.northwestern.edu
} 


\begin{abstract}
This paper addresses the problem of power and sample size calculation for a stepwise multiple test procedure (SD2PC) proposed in Tamhane, Dunnett, Green and Wetherington (2001) to identify the maximum safe dose of a compound. A general expression for the power of this procedure is derived. It is used to find the minimum overall power and minimum power under the constraint that the dose response function is bounded from below by a linear response function. It is shown that the two minima are attained under step and linear response functions, respectively. The sample sizes necessary on the zero dose control and each of the positive doses to guarantee a specified power requirement are calculated under these two least favorable configurations. A technique involving a continuous approximation to the sample sizes is used to reduce the number of quantities that need to be tabled, and to derive the asymptotically optimal allocation of the total sample size between the zero dose and the positive doses. An example is given to illustrate use of the tables. Extensions of the basic formulation are noted.
\end{abstract}

$M S C: 62 \mathrm{~J} 15,62 \mathrm{~K} 99$

Keywords: Design of dose response experiments; Multiple testing; Multivariate normal distribution; Toxicology 


\section{Introduction}

In Tamhane, Dunnett, Green and Wetherington (2001) (referred to as TDGW hereafter) we proposed three stepwise multiple test procedures (labeled there as SD1PC, SD2PC and $\mathrm{SD} 1 \mathrm{HC})$ to find the maximum safe dose (MAXSD) of a compound in relation to a zero dose control. These procedures control the type I familywise error rate (FWE), which is the probability of declaring any unsafe dose as safe, at a specified level $\alpha$. In TDGW we investigated the power properties of the proposed procedures via simulation. In this paper we derive analytical expressions for the power of SD2PC (which is a preferred procedure for the reasons explained in the sequel). We use these expressions to calculate the sample sizes necessary to guarantee a specified power requirement.

Practical applications of the MAXSD approach were described in TDGW. This problem arises in toxicology experiments to evaluate safety of agricultural compounds where efficacy is not a concern. The MAXSD approach was motivated in our collaborations with scientists from DuPont.

The outline of the paper is as follows. In Section 2 we define the notation and formulate the problem. The SD2PC procedure is briefly reviewed in Section 3. An expression for its power is derived in Section 4. This expression is used to find the minimum overall power and minimum power under the constraint that the dose response function is bounded from below by a linear response function. It is shown that the two minima are attained under step and linear response functions, respectively. The exact discrete optimization problem of finding the smallest total sample size under these two least favorable configurations to guarantee a specified power requirement is stated and solved in Section 5. A continuous approximation to this problem for the minimum overall power is stated and solved in Section 6. The solution from the continuous approximate problem is used as an initial solution for the numerical search of the corresponding exact discrete optimum. The continuous problem is also useful for deriving asymptotically optimal allocation of the sample sizes between the zero dose control and positive doses. An example is given in Section 7. Finally, some extensions of the basic formulation are noted in Section 8. An evaluation of the derivative of the power function needed in the continuous optimization problem is given in the Appendix. 


\section{Problem Formulation}

We assume the usual homoscedastic normal theory one-way layout model with increasing doses denoted by $0,1, \ldots, k$, where 0 is the zero dose (control). The unknown dose means are denoted by $\mu_{i}$ and the unknown common error variance by $\sigma^{2}$. A smaller $\mu_{i}$ (e.g., a lower yield of a crop contaminated by a herbicide) is assumed to represent a more toxic response. The case where a larger $\mu_{i}$ represents a more toxic response can be handled analogously.

We take $n_{i}$ observations, $y_{i j}\left(1 \leq j \leq n_{i}\right)$, on dose $i$ and compute the sample means $\bar{y}_{i}$ and the pooled sample variance $s^{2}$ based on $\nu=\sum_{i=0}^{k} n_{i}-(k+1)$ degrees of freedom (d.f.). The corresponding random variables $\bar{Y}_{i}$ and $S^{2}$ are distributed independently as $N\left(\mu_{i}, \sigma^{2} / n_{i}\right)$ and $\sigma^{2} \chi_{\nu}^{2} / \nu$, respectively.

We regard a dose as unsafe if it causes a decrease in mean yield below a specified percentage (say, 10\%) of the mean yield $\mu_{0}$ at the zero dose level. More generally, we regard the ith dose as unsafe if $\mu_{i} \leq \lambda \mu_{0}$ and as safe if $\mu_{i}>\lambda \mu_{0}$ where $\lambda<1$ is specified, e.g., $\lambda=0.90$ for a $10 \%$ decrease in the mean yield compared to $\mu_{0}$. The maximum safe dose (MAXSD) for specified $\lambda$ is defined as

$$
\operatorname{MAXSD}=\max \left\{i: \mu_{j}>\lambda \mu_{0} \forall j \leq i\right\}
$$

If a larger $\mu_{i}$ represents a more toxic response then $\lambda>1$. In that case, doses with $\mu_{i} \geq \lambda \mu_{0}$ are regarded as unsafe and those with $\mu_{i}<\lambda \mu_{0}$ are regarded as safe. The MAXSD is defined as MAXSD $=\max \left\{i: \mu_{j}<\lambda \mu_{0} \forall j \leq i\right\}$. Note that the above definition assumes that $\mu_{0}$ is $>0$. This is a reasonable assumption in most practical problems as the measurements are positive. If necessary, a preliminary test could be performed to check it.

We want to guarantee that the probability that any unsafe dose is declared safe is no more than a specified constant $\alpha$. If M $\widehat{A X S D}$ denotes the estimated MAXSD then this requirement translates to

$$
P\{\mathrm{MAXSD}>\operatorname{MAXSD}\} \leq \alpha
$$


Now consider the family of hypothesis testing problems

$$
H_{0 i}: \mu_{i} \leq \lambda \mu_{0} \text { vs. } H_{1 i}: \mu_{i}>\lambda \mu_{0} \quad(1 \leq i \leq k)
$$

Here $H_{0 i}$ states that the $i$-th dose is unsafe and $H_{1 i}$ states that the $i$-th dose is safe. After testing the hypotheses we set $\mathrm{MAXSD}=\max \left\{i: H_{0 i}\right.$ is rejected $\}$. The error probability requirement (2.2) is satisfied if we control the type I FWE at level $\alpha$ :

$$
\mathrm{FWE}=P\left\{\text { Any true } H_{0 i} \text { is rejected }\right\} \leq \alpha
$$

for the family of hypotheses (2.3).

It was shown in Section 8 of TDGW that this requirement is satisfied if the null hypotheses in (2.3) are tested in a step-down (SD) manner beginning with $H_{01}$; if it is rejected then $H_{02}$ and so on, each at level $\alpha$, which is what SD2PC does. On the other hand, SD1PC and SD1HC test $\bigcap_{j=i}^{k} H_{0 j} \subseteq H_{0 i}$ in a step-down manner, each at level $\alpha$. Under the assumption of monotonicity, $\mu_{0} \geq \mu_{1} \geq \cdots \geq \mu_{k}$, we have $H_{0 i}=\bigcap_{j=i}^{k} H_{0 j}$. Therefore SD1PC and SD1HC procedures also control (2.2). However, if the means are not monotone then only SD2PC controls this requirement as shown by Bauer (1997).

In the present paper we restrict attention to the SD2PC procedure because (i) as noted above, it is valid even if the dose response function is non-monotone, (ii) simulation studies reported in TDGW showed that SD2PC generally has high power, especially under the linear dose response function, although its power can be very low under the step response function when the MAXSD is high, (iii) it is very easy to apply and explain to a practitioner, and (iv) it can be readily extended to non-normal setups, where statistics such as Mann-Whitney may be used for comparing each dose with the zero dose control.

Specification of the power requirement for the hypothesis testing problem (2.3) entails specification of two constants, $\delta(0<\delta<1-\lambda)$ and $1-\beta(\alpha<1-\beta<1)$. In analogy with the definition (2.1) of MAXSD, we specify the power requirement as follows:

$$
P\left\{\text { Reject all false } H_{0 i} \text { with } \min _{j \leq i} \mu_{j} \geq(\lambda+\delta) \mu_{0}\right\} \geq 1-\beta \text {. }
$$


Under monotonicity, this is equivalent to a more stringent requirement in which $\min _{j \leq i} \mu_{j}$ is replaced by $\mu_{i}$. As an example, suppose $\lambda=0.90, \delta=0.05$ and $1-\beta=0.80$. Then any dose with mean $\mu_{i}>0.90 \mu_{0}$ is safe, but we want to guarantee that all consecutive doses with means $\mu_{i} \geq 0.95 \mu_{0}$ are declared safe with probability at least 0.80 .

We note that Horn and Vollandt (2002) have done sample size calculations for a similar setting but for a formulation that uses an additive threshold constant in contrast to our multiplicative constant $\lambda$.

\section{SD2PC Procedure}

The SD2PC procedure is a step-down (SD) testing procedure based on pairwise contrasts, $\bar{y}_{i}-\lambda \bar{y}_{0}(1 \leq i \leq k)$. The corresponding $t$-statistics are

$$
t_{i}=\frac{\bar{y}_{i}-\lambda \bar{y}_{0}}{s \sqrt{1 / n_{i}+\lambda^{2} / n_{0}}}(1 \leq i \leq k) .
$$

SD2PC uses an $\alpha$-level $t$-test at each step, beginning with the test of $H_{01}$. Thus it rejects $H_{01}$ if $t_{1}>t_{\nu, \alpha}$, where $t_{\nu, \alpha}$ is the upper $\alpha$ critical point of Student's $t$ with $\nu$ d.f., and goes on to test $H_{02}$. If $H_{01}$ is not rejected then testing stops and it is concluded that M $\widehat{\mathrm{AXSD}}<1$, i.e., dose 1 itself is not proven safe. In general, if $H_{01}, H_{02}, \ldots, H_{0 i}$ have been tested and rejected and if $i<k$ then $H_{0, i+1}$ is tested next and rejected if $t_{i+1}>t_{\nu, \alpha}$; otherwise testing stops and it is concluded that $\mathrm{MAXSD}=i$.

\section{Power of SD2PC Procedure}

Suppose that $\mu_{i} \geq(\lambda+\delta) \mu_{0}$ for $1 \leq i \leq m$ and $\mu_{i}<(\lambda+\delta) \mu_{0}$ for $i>m$, so that effectively $m$ is the MAXSD as far as the power requirement (2.5) is concerned, which is then

$$
P\left\{\text { Reject } H_{0 i} \text { for } i=1,2, \ldots, m\right\} \geq 1-\beta \text {. }
$$

To write the power expression, introduce the following notation:

$$
Z_{i}=\frac{\bar{Y}_{i}-\lambda \bar{Y}_{0}-\left(\mu_{i}-\lambda \mu_{0}\right)}{\sigma \sqrt{1 / n_{i}+\lambda^{2} / n_{0}}}(1 \leq i \leq k) \text { and } U=\frac{S}{\sigma}
$$


Further let

$$
\lambda_{i}=\frac{\mu_{i}}{\mu_{0}} \text { and } r_{i}=\frac{n_{0}}{n_{i}}(1 \leq i \leq k) .
$$

Then $Z_{1}, Z_{2}, \ldots, Z_{k}$ have a $k$-variate standard normal distribution with correlations

$$
\rho_{i j}=\operatorname{Corr}\left(Z_{i}, Z_{j}\right)=\frac{\lambda^{2}}{\sqrt{\left(r_{i}+\lambda^{2}\right)\left(r_{j}+\lambda^{2}\right)}}=\tau_{i} \tau_{j} \quad(1 \leq i \neq j \leq k)
$$

where

$$
\tau_{i}=\frac{\lambda}{\sqrt{r_{i}+\lambda^{2}}}(1 \leq i \leq k)
$$

and $U=S / \sigma \sim \sqrt{\chi_{\nu}^{2} / \nu}$ independent of the $Z_{i}$ 's. In other words, $T_{i}=Z_{i} / U(1 \leq i \leq k)$ have a central $k$-variate $t$-distribution with the above correlation structure and $\nu$ d.f. Then, for fixed $m(1 \leq m \leq k)$, the power expression (4.1) is given by

$$
\begin{aligned}
& P\left\{\frac{\bar{Y}_{i}-\lambda \bar{Y}_{0}}{S \sqrt{1 / n_{i}+\lambda^{2} / n_{0}}}>t_{\nu, \alpha}(1 \leq i \leq m)\right\} \\
= & P\left\{\frac{Z_{i}}{U}>t_{\nu, \alpha}-\frac{\mu_{i}-\lambda \mu_{0}}{S \sqrt{1 / n_{i}+\lambda^{2} / n_{0}}}(1 \leq i \leq m)\right\} \\
= & P\left\{Z_{i}>t_{\nu, \alpha} U-\frac{\left(\lambda_{i}-\lambda\right)\left(\mu_{0} / \sigma\right)}{\sqrt{1 / n_{i}+\lambda^{2} / n_{0}}}(1 \leq i \leq m)\right\} \\
= & \int_{0}^{\infty} P\left\{-Z_{i} \leq-t_{\nu, \alpha} u+\frac{\left(\lambda_{i}-\lambda\right)\left(\mu_{0} / \sigma\right)}{\sqrt{1 / n_{i}+\lambda^{2} / n_{0}}}(1 \leq i \leq m)\right\} h_{\nu}(u) d u,
\end{aligned}
$$

where

$$
h_{\nu}(u)=\frac{2(\nu / 2)^{\nu / 2}}{\Gamma(\nu / 2)} u^{\nu-1} \exp \left(-\nu u^{2} / 2\right), \quad u \geq 0
$$

denotes the p.d.f. of $U \sim \sqrt{\chi_{\nu}^{2} / \nu}$. Put

$$
c_{i}(u)=-t_{\nu, \alpha} u+\frac{\left(\lambda_{i}-\lambda\right)\left(\mu_{0} / \sigma\right)}{\sqrt{1 / n_{i}+\lambda^{2} / n_{0}}}(1 \leq i \leq m) .
$$

Then noting that the $-Z_{i}$ 's have the same joint distribution as the $Z_{i}$ 's, and by exploiting the product correlation structure in (4.2), we can express the $m$-variate normal probability 
in the last step of (4.3) as a univariate iterated integral (see equation (1.1a) in Appendix 3 of Hochberg and Tamhane (1987)) leading to the following expression for power:

$$
\int_{0}^{\infty}\left\{\int_{-\infty}^{\infty} \prod_{i=1}^{m} \Phi\left[\frac{\tau_{i} z+c_{i}(u)}{\sqrt{1-\tau_{i}^{2}}}\right] \phi(z) d z\right\} h_{\nu}(u) d u
$$

where $\Phi(\cdot)$ and $\phi(\cdot)$ are, respectively, the c.d.f. and the p.d.f. of the standard normal distribution.

\subsection{Minimum Overall Power}

From symmetry considerations, we will assume that the experiment is designed with $n_{1}=n_{2}=\cdots=n_{k}=n$ (say). Let $n_{0} / n=r$. Suppose that the dose response function satisfies

$$
\mu_{i} \geq(\lambda+\delta) \mu_{0}, 1 \leq i \leq m \text { and } \mu_{m+1}<(\lambda+\delta) \mu_{0}
$$

Then, since (4.4) is increasing in the $\lambda_{i}$, its minimum for fixed $m(1 \leq m \leq k)$ is attained when $\mu_{i}=(\lambda+\delta) \mu_{0}$ for $i=1, \ldots, m$. Thus the step response function is least favorable. Furthermore, the minimum over $m$ is attained when $m=k$ and it depends on $\delta$ and $\mu_{0} / \sigma$ only through their product, denoted by $\eta=\delta\left(\mu_{0} / \sigma\right)$. This minimum power is given by

$$
P_{k}\left(n_{0}, n, \lambda, \eta\right)=\int_{0}^{\infty}\left\{\int_{-\infty}^{\infty} \Phi^{k}\left[\frac{\tau z+c(u)}{\sqrt{1-\tau^{2}}}\right] \phi(z) d z\right\} h_{\nu}(u) d u
$$

where we have put

$$
c(u)=-t_{\nu, \alpha} u+\frac{\eta}{\sqrt{1 / n+\lambda^{2} / n_{0}}}, \quad \text { and } \tau=\frac{\lambda}{\sqrt{r+\lambda^{2}}} .
$$

\subsection{Minimum Power Under Linear Lower Bound on the Dose Response Function}

Suppose that the dose response function is bounded below by a linear function:

$$
\mu_{i} \geq \mu_{0}-i \xi(1 \leq i \leq k)
$$

for some $\xi>0$. For fixed $m(1 \leq m \leq k)$ consider the restricted parameter space $\Omega=$ 
$\bigcup_{m=1}^{k} \Omega_{m}$, where

$$
\Omega_{m}=\left\{\boldsymbol{\mu}=\left(\mu_{0}, \mu_{1}, \ldots, \mu_{k}\right): \mu_{i} \geq \mu_{0}-i \xi \geq(\lambda+\delta) \mu_{0}(1 \leq i \leq m), \mu_{m+1}<(\lambda+\delta) \mu_{0}\right\}
$$

It is readily seen that for $\boldsymbol{\mu} \in \Omega_{m}$, the power (4.3) is minimized when $\mu_{i}=\mu_{0}-i \xi$ and $\xi$ is as large as possible, i.e.,

$$
\xi=\frac{\{1-(\lambda+\delta)\} \mu_{0}}{m}
$$

(The values of $\mu_{i}$ for $i>m$ are irrelevant.) Thus the linear response function is least favorable in this case. Then the power expression becomes

$$
\begin{aligned}
& P\left\{-Z_{i} \leq-t_{\nu, \alpha} U+\frac{\{(1-\lambda)-[1-(\lambda+\delta)](i / m)\}\left(\mu_{0} / \sigma\right)}{\sqrt{1 / n+\lambda^{2} / n_{0}}}(1 \leq i \leq m)\right\} \\
= & \int_{0}^{\infty}\left\{\int_{-\infty}^{\infty} \prod_{i=1}^{m} \Phi\left[b_{i, m}(z, u)\right] \phi(z) d z\right\} h_{\nu}(u) d u,
\end{aligned}
$$

where

$$
b_{i, m}(z, u)=\frac{\tau z+c_{i, m}(u)}{\sqrt{1-\tau^{2}}}(1 \leq i \leq m)
$$

and

$$
c_{i, m}(u)=-t_{\nu, \alpha} u+\frac{\{(1-\lambda)-[1-(\lambda+\delta)](i / m)\}\left(\mu_{0} / \sigma\right)}{\sqrt{1 / n+\lambda^{2} / n_{0}}}(1 \leq i \leq m) .
$$

Since $i / m \leq(i+1) /(m+1)$, it follows that for every fixed $(z, u), b_{i, m}(z, u) \geq b_{i+1, m+1}(z, u)$. Hence

$$
\prod_{i=1}^{m} \Phi\left[b_{i, m}(z, u)\right] \geq \prod_{i=1}^{m} \Phi\left[b_{i+1, m+1}(z, u)\right]=\prod_{i=2}^{m+1} \Phi\left[b_{i, m+1}(z, u)\right] \geq \prod_{i=1}^{m+1} \Phi\left[b_{i, m+1}(z, u)\right]
$$

Thus (4.7) is minimized when $m=k$. The final minimum power expression for the linear response function depends on both $\delta$ and $\left(\mu_{0} / \sigma\right)$, and is given by

$$
P_{k}\left(n_{0}, n, \lambda, \delta, \mu_{0} / \sigma\right)=\int_{0}^{\infty}\left\{\int_{-\infty}^{\infty} \prod_{i=1}^{k} \Phi\left[\frac{\tau z+c_{i, k}(u)}{\sqrt{1-\tau^{2}}}\right] \phi(z) d z\right\} h_{\nu}(u) d u
$$


where $\tau$ is as defined in (4.6) and

$$
c_{i, k}(u)=-t_{\nu, \alpha} u+\frac{\{(1-\lambda)-[1-(\lambda+\delta)](i / k)\}\left(\mu_{0} / \sigma\right)}{\sqrt{1 / n+\lambda^{2} / n_{0}}}(1 \leq i \leq k) .
$$

To evaluate (4.8), the quantity $\mu_{0} / \sigma$, which is the inverse of the coefficient of variation for the zero dose, needs to be specified or at least a lower bound on it. (To evaluate (4.5), only the product $\eta=\delta\left(\mu_{0} / \sigma\right)$ needs to be specified.) The larger the coefficient of variation, the smaller the power and hence the larger the sample size needed to guarantee a specified power requirement.

\section{Exact Discrete Optimization Problem}

The exact optimization problem to be solved is the following: For given $k$ and specified $\lambda, \delta,\left(\mu_{0} / \sigma\right), \alpha$ and $1-\beta$,

$$
\text { Minimize } N=n_{0}+k n \text { subject to }(4.5) \text { or }(4.8) \geq 1-\beta \text {. }
$$

As noted before, in case of the overall minimum power given by (4.5), one need not specify $\delta$ and $\left(\mu_{0} / \sigma\right)$ separately, but only $\eta=\delta\left(\mu_{0} / \sigma\right)$. One can use numerical search to solve this optimization problem. However, it can be very time consuming and laborious. Numerical search can be accelerated if we know the optimum value of the ratio $r=n_{0} / n$ because then we only need to find the smallest $N$ so that the expression (4.5) (or (4.8)) is $\geq 1-\beta$. This is an easy trial and error exercise because the minimum power is a strictly increasing function of $N$.

In the next section we find the optimum value of $r$ by solving an approximate continuous problem for the overall minimum power case. (The constrained minimum under a linear response lower bound case could be studied analogously, but it is analytically far more involved.) This continuous approximation also has the advantage that it obviates the need to compute $N$ (and associated $n_{0}$ and $n$ ) for each specified value of $\eta=\delta\left(\mu_{0} / \sigma\right)$; rather it is only necessary to compute a quantity $\gamma$ defined in (6.1) and the associated $r$. We are also able to study the asymptotic behavior of optimum $r$ and show that $r \rightarrow \lambda \sqrt{k}$ 
(which is a simple extension of Dunnett's (1955) square root allocation rule) as $\gamma \rightarrow \infty$. The optimum $r$ found by solving the approximate continuous problem is used as a starting solution for numerical search to find the exact discrete optimum solution to the optimization problem. The corresponding optimum sample sizes are given in Tables 1 and 2 for $k=$ $3,4,5,6,1-\beta=0.70,0.80,0.90, \mu_{0} / \sigma=5,10, \lambda=0.75,0.80,0.85,0.90, \delta=0.05$ (which corresponds to $\eta=0.25,0.50)$ and $\alpha=0.05$. These tables are self-explanatory. Their use is illustrated in Section 7.

\section{Approximate Continuous Optimization Problem}

To simplify the optimization problem we will assume $\nu=\infty$ so that the power expression (4.5) for step response reduces to a single integral. Furthermore, we define the quantity

$$
\gamma=\eta \sqrt{N}=\left(\frac{\delta \mu_{0}}{\sigma}\right) \sqrt{N}
$$

which we treat as a continuous variable along with $r$. Thus the optimization problem for the step response case can be stated as: For given $k$ and specified $\lambda, \alpha$ and $1-\beta$, find the smallest value of $\gamma$ and the associated value of $r$ so that

$$
\int_{-\infty}^{\infty} \Phi^{k}\left[\frac{\tau z+c}{\sqrt{1-\tau^{2}}}\right] \phi(z) d z=1-\beta
$$

where

$$
c=-z_{\alpha}+\frac{\gamma \sqrt{r}}{\sqrt{(k+r)\left(r+\lambda^{2}\right)}} \text { and } \tau=\frac{\lambda}{\sqrt{r+\lambda^{2}}}
$$

here $z_{\alpha}=t_{\infty, \alpha}$ is the upper $\alpha$ critical point of the standard normal distribution.

To solve this problem, first fix $r$ and solve (6.2) for $c$ or equivalently $\gamma$. An explicit solution for $c$ can be obtained as follows. Note that the integral in (6.2) equals $P\left\{Z_{1} \leq\right.$ $\left.c, Z_{2} \leq c, \ldots, Z_{k} \leq c\right\}$, where $Z_{1}, Z_{2}, \ldots, Z_{k}$ have a $k$-variate standard normal distribution with common correlation $\tau^{2}=\lambda^{2} /\left(r+\lambda^{2}\right)$. Therefore $c$ is the upper $\beta$ critical point of $\max _{1 \leq i \leq k} Z_{i}$; we denote this critical point by $z_{k, \tau^{2}, \beta}$. Dunnett's (1989) program can be used 
to calculate $c=z_{k, \tau^{2}, \beta}$. Then

$$
\gamma=\eta \sqrt{N}=\left(z_{\alpha}+z_{k, \tau^{2}, \beta}\right) \sqrt{\frac{(k+r)\left(r+\lambda^{2}\right)}{r}},
$$

and the integral in (6.2) is a function of $r$ alone. We denote it by $\psi(r)$, and find the optimum value of $r$ by setting

$$
\psi^{\prime}(r)=\frac{d \psi(r)}{d r}=0
$$

In Appendix we show, using the same methods as in Bechhofer (1969), that

$$
\begin{aligned}
\psi^{\prime}(r)= & -\frac{\lambda k(k-1) \tau\left(1-\tau^{2}\right) r^{-3 / 2}}{\sqrt{8 \pi\left(1+\tau^{2}\right)}} \phi\left(c \sqrt{\frac{2}{1+\tau^{2}}}\right) \Phi_{k-2}\left(c \sqrt{\left.\frac{1-\tau^{2}}{\left(1+\tau^{2}\right)\left(1+2 \tau^{2}\right)} \mid \frac{1+\tau^{2}}{1+2 \tau^{2}}\right)}\right. \\
& +\frac{k \sqrt{1-\tau^{2}}}{2 \tau}\left[\tau d-\lambda c\left(1-\tau^{2}\right) r^{-3 / 2}\right] \phi(c) \Phi_{k-1}\left(c \sqrt{\frac{1-\tau^{2}}{1+\tau^{2}}} \mid \frac{\tau^{2}}{1+\tau^{2}}\right),
\end{aligned}
$$

where $\Phi_{k}(x \mid \rho)$ denotes the equicoordinate c.d.f. at point $x$ of a $k$-variate standard normal distribution with common correlation $\rho$, and

$$
d=\frac{\lambda c r^{-3 / 2}}{\tau}+z_{\alpha} \frac{\lambda^{2}}{r^{2}}\left(1+\frac{\lambda^{2}}{r}\right)^{-1 / 2}-\gamma(k+r)^{-3 / 2} .
$$

The solution to the equation $\psi^{\prime}(r)=0$ is substituted in $\tau=\lambda / \sqrt{r+\lambda^{2}}$ to find a new value of $c=z_{k, \tau^{2}, \beta}$ and the process is iterated until convergence is reached. The solutions to the continuous optimization problem are given in Table 3 for the same values of $k, \lambda, 1-\beta$ and $\alpha$ as in Tables 1 and 2. Use of this table is illustrated in Section 7.

It may be noted from Table 3 that as $\gamma=\eta \sqrt{N}$ increases, $r$ approaches $\lambda \sqrt{k}$. This fact can be shown analytically as follows. As $\gamma$ and $c \rightarrow \infty$, the first term in the expression for $\psi^{\prime}(r)$ goes to zero faster than the second term because $c \sqrt{2 /\left(1+\tau^{2}\right)}<c$ and hence $\phi\left(c \sqrt{2 /\left(1+\tau^{2}\right)}\right)>\phi(c)$. As a result, the second term is the dominant one. Therefore, for large $c$, the solution to the equation $\psi^{\prime}(r)=0$ can be approximated by the solution to the equation

$$
\tau d-\lambda c\left(1-\tau^{2}\right) r^{-3 / 2}=0
$$


where $d$ is given by (6.4). Now for large $c$ and hence large $\gamma$,

$$
d \approx \frac{\lambda c r^{-3 / 2}}{\tau}-\gamma(k+r)^{-3 / 2} \text { and } c \approx \frac{\gamma \sqrt{r}}{\sqrt{(k+r)\left(r+\lambda^{2}\right)}}
$$

Substituting these approximations and $\tau=\lambda / \sqrt{r+\lambda^{2}}$ in (6.5), we obtain

$$
\begin{aligned}
& \frac{\lambda \gamma}{r \sqrt{(k+r)\left(r+\lambda^{2}\right)}}-\frac{\lambda \gamma(k+r)^{-3 / 2}}{\sqrt{r+\lambda^{2}}}-\frac{\lambda \gamma}{\sqrt{(k+r)\left(r+\lambda^{2}\right)^{3}}} \\
= & \frac{\lambda \gamma}{\sqrt{(k+r)\left(r+\lambda^{2}\right)}}\left[\frac{1}{r}-\frac{1}{k+r}-\frac{1}{\lambda^{2}+r}\right] \\
= & 0,
\end{aligned}
$$

the solution to which can be easily checked to be $r=\lambda \sqrt{k}$.

This approximately optimum value of $r$ can be shown to minimize

$$
\operatorname{Var}\left(\bar{Y}_{i}-\lambda \bar{Y}_{0}\right)=\sigma^{2}\left(\frac{1}{n}+\frac{\lambda^{2}}{n_{0}}\right)
$$

Putting $n_{0}=r n$ and hence $n=N /(k+r)$, we see that, for fixed total sample size $N$, we need to minimize $(k+r)\left(1+\lambda^{2} / r\right)$, and the minimizing value is $r=\lambda \sqrt{k}$.

\section{Example}

Suppose that $k=5$ doses are to be compared to a zero dose control to find the MAXSD. The following quantities are specified: $\lambda=0.90, \delta=0.05, \alpha=0.05,1-\beta=0.70$ and $\mu_{0} / \sigma=10$. Then $\eta=(0.05)(10)=0.50$. Then from Table 1 , the exact discrete optimum sample sizes are found to be $n_{0}=76, n=53$ and $N=341$. Let us see how these sample sizes compare with those computed from Table 3. For $k=5, \lambda=0.80, \alpha=0.05,1-\beta=0.70$, we find that $\gamma=9.190$ and $r=1.429$. Therefore

$$
N=\left(\frac{\gamma}{\eta}\right)^{2}=\left(\frac{9.190}{0.50}\right)^{2}=338
$$

which gives

$$
n=\frac{N}{k+r}=\frac{338}{5+1.429}=53 \text { and } n_{0}=N-k n=73 .
$$


We see that these sample sizes are quite close to those obtained from Table 1, but are slightly smaller. This will generally be the case because the continuous approximation assumes that $\sigma$ is known which results in slightly smaller sample sizes. However, the differences are not appreciable especially if $N$ is large.

For the linear response case, from Table 2 we find that $n_{0}=43, n=28$ and $N=183$. Note that the sample sizes are much smaller in this case.

\section{Extensions}

In Section 4.2 we assumed that $\mu_{i} \geq \mu_{0}-i \xi$. This linear lower bound implicitly assumes that the doses $d_{i}$ are equispaced. A more general approach might be to incorporate the actual dose values in this lower bound, e. g., assume that

$$
\mu_{i} \geq \mu_{0}-\left(d_{i}-d_{0}\right) \xi(1 \leq i \leq k)
$$

where $\xi>0$. We refer to this lower bound as the generalized linear response. Alternatively, one could assume an exponential response lower bound:

$$
\mu_{i} \geq \mu_{0}(1 / \xi)^{d_{i}-d_{0}} \Longleftrightarrow \ln \mu_{i} \geq \ln \mu_{0}-\left(d_{i}-d_{0}\right) \ln \xi(1 \leq i \leq k),
$$

where $\xi \geq 1$.

First consider the generalized linear response case. The definitions of $\Omega_{m}$ and $\Omega$ can be modified in an obvious manner. Then for $\boldsymbol{\mu} \in \Omega_{m}$, the power is minimized when

$$
\xi=\frac{[1-(\lambda+\delta)] \mu_{0}}{d_{m}-d_{0}}
$$

If the sequence $\left\{d_{i}-d_{0}, i=1,2, \ldots, k\right\}$ is log-concave, i.e., if $\left(d_{i}-d_{0}\right) /\left(d_{i+1}-d_{0}\right)$ is increasing in $i$, then the minimum power is given by (4.7), where $i / k$ in the definition of $c_{i, k}(u)$ must be replaced by $\left(d_{i}-d_{0}\right) /\left(d_{k}-d_{0}\right)$.

For the exponential case, $\Omega_{m}$ is given by

$$
\Omega_{m}=\left\{\left(\mu_{0}, \mu_{1}, \ldots, \mu_{k}\right): \mu_{i} \geq \mu_{0}(1 / \xi)^{d_{i}-d_{0}}(1 \leq i \leq m), \mu_{m+1}<(\lambda+\delta) \mu_{0}\right\} .
$$


The power can be shown to be minimized when $1 / \xi=(\lambda+\delta)^{1 /\left(d_{m}-d_{0}\right)}$. Again, if the sequence $\left\{d_{i}-d_{0}, i=1,2, \ldots, k\right\}$ is log-concave then the minimum power is given by (4.7) with

$$
\left.c_{i, k}(u)=-t_{\nu, \alpha}+\left\{\left[(\lambda+\delta)^{\frac{d_{i}-d_{0}}{d_{k}-d_{0}}}-\lambda\right]\left(\mu_{0} / \sigma\right)\right]\right\} / \sqrt{1 / n+\lambda^{2} / n_{0}} .
$$

Power and sample size calculations can be done under these dose response functions by simple modifications in our computer programs. We can provide the programs to anyone interested.

This paper has given a method to compute the sample size for the SD2PC step-down multiple test procedure for finding the maximum safe dose of a compound. The method is based on the least favorable configuration approach. Therefore the resulting sample sizes may be too conservative for practical applications. To resolve this problem, a Bayesian approach can be adopted by putting prior distributions on the unknown parameters. However, this approach faces analytical difficulties as well as the fact that adequate previous knowledge is often lacking to specify full priors. A way out of both these difficulties is to specify a discrete prior distribution on the MAXSD itself, i.e., one could specify prior probabilities $p_{1}, p_{2}, \ldots, p_{k}$, where $p_{m}$ is the probability that the $m$ th dose is MAXSD $(1 \leq m \leq k)$. Then instead of using the power expressions (4.5) or (4.7), which use MAXSD $=k$ as the LFC, we will obtain the weighted averages of the corresponding expressions for different values of MAXSD. The simplest prior is the uniform prior: $p_{1}=p_{2}=\cdots=p_{k}=1 / k$. We plan to use this uniform prior to compute the required sample sizes and compare them with the sample sizes reported here using the LFC approach. These calculations will be reported in a future paper.

Acknowledgments: This research was partially supported by an educational grant from DuPont. We thank Dr. John Green of DuPont Safety, Health and Environmental Excellence Center, Haskell Laboratory for Toxicology and Industrial Medicine for providing data that guided our computations of the tables and Professor Charles Dunnett for some help with computations. We thank a referee for help in improving the paper. 


\section{Appendix: Evaluation of the Derivative $\psi^{\prime}(r)$}

For $\nu=\infty$ the minimum power expression, $\psi(r)$, for the step response function is given by (6.2). Taking its derivative with respect to $r$, we get

$$
\psi^{\prime}(r)=k \int_{-\infty}^{\infty} \Phi^{k-1}\left[\frac{\tau z+c}{\sqrt{1-\tau^{2}}}\right]\left[\frac{d}{d r}\left(\frac{\tau z+c}{\sqrt{1-\tau^{2}}}\right)\right] \phi\left(\frac{\tau z+c}{\sqrt{1-\tau^{2}}}\right) \phi(z) d z
$$

First calculate,

$$
\begin{aligned}
\frac{d}{d r}\left(\frac{\tau z+c}{\sqrt{1-\tau^{2}}}\right) & =\frac{d}{d r}\left(\frac{\lambda z / \sqrt{r+\lambda^{2}}-z_{\alpha}+\delta \gamma \sqrt{r /(k+r)\left(r+\lambda^{2}\right)}}{\sqrt{1-\lambda^{2} /\left(r+\lambda^{2}\right)}}\right) \\
& =\frac{d}{d r}\left(\frac{\lambda z-z_{\alpha} \sqrt{r+\lambda^{2}}+\delta \gamma \sqrt{r /(k+r)}}{\sqrt{r}}\right) \\
& =\frac{d}{d r}\left(\frac{\lambda z}{\sqrt{r}}-z_{\alpha} \sqrt{1+\frac{\lambda^{2}}{r}}+\frac{\delta \gamma}{\sqrt{k+r}}\right) \\
& =-\frac{1}{2} \lambda z r^{-3 / 2}+\frac{1}{2} z_{\alpha}\left(1+\frac{\lambda^{2}}{r}\right)^{-1 / 2} \lambda^{2} r^{-2}-\frac{1}{2} \delta \gamma(k+r)^{-3 / 2} .
\end{aligned}
$$

Put

$$
y=\frac{\tau z+c}{\sqrt{1-\tau^{2}}}
$$

Then the above expression becomes

$$
\frac{d}{d r}\left(\frac{\tau z+c}{\sqrt{1-\tau^{2}}}\right)=-\frac{\lambda r^{-3 / 2} \sqrt{1-\tau^{2}}}{2 \tau} y+\frac{\lambda c r^{-3 / 2}}{2 \tau}+\frac{1}{2} z_{\alpha}\left(1+\frac{\lambda^{2}}{r}\right)^{-1 / 2} \lambda^{2} r^{-2}-\frac{1}{2} \delta \gamma(k+r)^{-3 / 2} .
$$

Substitute this expression in $\psi^{\prime}(r)$ to obtain

$$
\begin{aligned}
\psi^{\prime}(r)= & k \int_{-\infty}^{\infty} \Phi^{k-1}(y) \phi(y) \phi^{*}(y) \frac{\sqrt{1-\tau^{2}}}{\tau} \\
& \times\left[-\frac{\lambda r^{-3 / 2} \sqrt{1-\tau^{2}}}{2 \tau} y+\frac{\lambda c r^{-3 / 2}}{2 \tau}+\frac{1}{2} z_{\alpha}\left(1+\frac{\lambda^{2}}{r}\right)^{-1 / 2} \lambda^{2} r^{-2}-\frac{1}{2} \delta \gamma(k+r)^{-3 / 2}\right] d y \\
= & -\frac{\lambda k r^{-3 / 2}\left(1-\tau^{2}\right)}{2 \tau^{2}} A \\
& +\frac{k \sqrt{1-\tau^{2}}}{2 \tau}\left\{\frac{\lambda c r^{-3 / 2}}{\tau}+z_{\alpha}\left(1+\frac{\lambda^{2}}{r}\right)^{-1 / 2} \lambda^{2} r^{-2}-\delta \gamma(k+r)^{-3 / 2}\right\} B,
\end{aligned}
$$


where

$\phi^{*}(y)=\phi\left(\frac{y \sqrt{1-\tau^{2}}-c}{\tau}\right), A=\int_{-\infty}^{\infty} y \Phi^{k-1}(y) \phi(y) \phi^{*}(y) d y$ and $B=\int_{-\infty}^{\infty} \Phi^{k-1}(y) \phi(y) \phi^{*}(y) d y$.

We now evaluate $A$ and $B$. Integrating by parts in $A$ with $u=\Phi^{k-1}(y) \phi^{*}(y)$ and $d v=$ $y \phi(y) d y$, we find that

$$
A=-\frac{1-\tau^{2}}{\tau^{2}} A+\frac{c \sqrt{1-\tau^{2}}}{\tau^{2}} B+(k-1) C
$$

where

$$
C=\int_{-\infty}^{\infty} \Phi^{k-2}(y) \phi^{2}(y) \phi^{*}(y) d y
$$

So,

$$
A=c \sqrt{1-\tau^{2}} B+\tau^{2}(k-1) C
$$

Notice that

$$
\begin{aligned}
\phi(y) \phi^{*}(y) & =\frac{1}{\sqrt{2 \pi}} e^{-y^{2} / 2} \frac{1}{\sqrt{2 \pi}} e^{-\left(y \sqrt{1-\tau^{2}}-c\right)^{2} / 2 \tau^{2}} \\
& =\phi(c) \phi\left(\frac{y-c \sqrt{1-\tau^{2}}}{\tau}\right)
\end{aligned}
$$

and

$$
\phi^{2}(y) \phi^{*}(y)=\frac{1}{\sqrt{2 \pi}} \phi\left(c \sqrt{\frac{2}{1+\tau^{2}}}\right) \phi\left(\frac{\sqrt{1+\tau^{2}}}{\tau} y-\frac{c}{\tau} \sqrt{\frac{1-\tau^{2}}{1+\tau^{2}}}\right) .
$$

Using the above relations we get

$$
B=\phi(c) \int_{-\infty}^{\infty} \Phi^{k-1}(y) \phi\left(\frac{y-c \sqrt{1-\tau^{2}}}{\tau}\right) d y
$$

Let

$$
x=\frac{y-c \sqrt{1-\tau^{2}}}{\tau} \Rightarrow y=\tau x+c \sqrt{1-\tau^{2}} \text { and } d y=\tau d x
$$

Then

$$
B=\phi(c) \tau \int_{-\infty}^{\infty} \Phi^{k-1}\left(\tau x+c \sqrt{1-\tau^{2}}\right) \phi(x) d x
$$




$$
=\phi(c) \tau \Phi_{k-1}\left(c \sqrt{\frac{1-\tau^{2}}{1+\tau^{2}}} \mid \frac{\tau^{2}}{1+\tau^{2}}\right)
$$

and

$$
C=\frac{1}{\sqrt{2 \pi}} \phi\left(c \sqrt{\frac{2}{1+\tau^{2}}}\right) \frac{\tau}{\sqrt{1+\tau^{2}}} \Phi_{k-2}\left(c \sqrt{\frac{1-\tau^{2}}{\left(1+\tau^{2}\right)\left(1+2 \tau^{2}\right)}} \mid \frac{\tau^{2}}{1+2 \tau^{2}}\right) .
$$

Combining the expressions for $A, B$ and $C$, and substituting them in the last expression for $\psi^{\prime}(r)$ we get the final expression (6.3).

\section{REFERENCES}

Bauer, P., 1997. A note on multiple testing procedures for dose finding, Biometrics, 53, $1125-1128$.

Bechhofer, R. E., 1969. Optimal allocation of observations when comparing several treatments with a control, Multivariate Analysis II (ed. P. R. Krishnaiah), New York: Academic Press, 465-473.

Dunnett, C. W., 1955. A multiple comparison procedure for comparing several treatments with a control, J. Amer. Statist. Assoc., 50, 1096 -1121.

Hochberg, Y. and Tamhane, A. C., 1987. Multiple Comparison Procedures, New York: John Wiley and Sons.

Horn, M. and Vollandt, R., 2002. Sample sizes for determining the minimum effective dose or the maximum safe dose in monotone dose-response relationships, Unpublished manuscript.

Tamhane, A. C., Dunnett, C. W., Green, J. and Wetherington, J., 2001. Multiple test procedures for identifying the maximum safe dose, J. Amer. Statist. Assoc., 96, 835843. 
Table 1: Exact (Discrete) Optimum Sample Sizes for Step Response $(\alpha=0.05)$

\begin{tabular}{|c|c|c|c|c|c|c|c|c|c|c|c|c|c|c|c|}
\hline$k$ & $1-\beta$ & $\eta$ & $\lambda$ & $N$ & $n_{0}$ & $n$ & $r$ & $k$ & $1-\beta$ & $\eta$ & $\lambda$ & $N$ & $n_{0}$ & $n$ & $r$ \\
\hline \multirow[t]{24}{*}{3} & \multirow[t]{8}{*}{0.70} & \multirow[t]{4}{*}{0.25} & 0.75 & 762 & 210 & 184 & 1.141 & \multirow[t]{24}{*}{5} & \multirow[t]{8}{*}{0.70} & \multirow[t]{4}{*}{0.25} & 0.75 & 1318 & 283 & 207 & 1.367 \\
\hline & & & 0.80 & 790 & 229 & 187 & 1.225 & & & & 0.80 & 1357 & 302 & 211 & 1.431 \\
\hline & & & 0.85 & 819 & 246 & 191 & 1.288 & & & & 0.85 & 1397 & 327 & 214 & 1.528 \\
\hline & & & 0.90 & 848 & 263 & 195 & 1.349 & & & & 0.90 & 1437 & 347 & 218 & 1.592 \\
\hline & & \multirow{4}{*}{0.50} & 0.75 & 192 & 54 & 46 & 1.174 & & & \multirow[t]{4}{*}{0.50} & 0.75 & 331 & 71 & 52 & 1.365 \\
\hline & & & 0.80 & 199 & 58 & 47 & 1.234 & & & & 0.80 & 341 & 76 & 53 & 1.434 \\
\hline & & & 0.85 & 206 & 62 & 48 & 1.292 & & & & 0.85 & 351 & 81 & 54 & 1.500 \\
\hline & & & 0.90 & 213 & 66 & 49 & 1.347 & & & & 0.90 & 361 & 91 & 54 & 1.685 \\
\hline & \multirow[t]{8}{*}{0.80} & \multirow[t]{4}{*}{0.25} & 0.75 & 917 & 260 & 219 & 1.187 & & \multirow[t]{8}{*}{0.80} & \multirow[t]{4}{*}{0.25} & 0.75 & 1545 & 350 & 239 & 1.464 \\
\hline & & & 0.80 & 952 & 283 & 223 & 1.269 & & & & 0.80 & 1592 & 377 & 243 & 1.551 \\
\hline & & & 0.85 & 987 & 303 & 228 & 1.329 & & & & 0.85 & 1640 & 405 & 247 & 1.640 \\
\hline & & & 0.90 & 1023 & 327 & 232 & 1.409 & & & & 0.90 & 1689 & 434 & 251 & 1.729 \\
\hline & & \multirow[t]{4}{*}{0.50} & 0.75 & 231 & 66 & 55 & 1.200 & & & \multirow[t]{4}{*}{0.50} & 0.75 & 388 & 88 & 60 & 1.467 \\
\hline & & & 0.80 & 240 & 72 & 56 & 1.286 & & & & 0.80 & 400 & 95 & 61 & 1.557 \\
\hline & & & 0.85 & 248 & 77 & 57 & 1.351 & & & & 0.85 & 412 & 102 & 62 & 1.645 \\
\hline & & & 0.90 & 258 & 84 & 58 & 1.448 & & & & 0.90 & 425 & 110 & 63 & 1.746 \\
\hline & \multirow[t]{8}{*}{0.90} & \multirow[t]{4}{*}{0.25} & 0.75 & 1159 & 337 & 274 & 1.230 & & \multirow[t]{8}{*}{0.90} & 0.25 & 0.75 & 1896 & 451 & 289 & 1.561 \\
\hline & & & 0.80 & 1204 & 367 & 279 & 1.315 & & & & 0.80 & 1957 & 487 & 294 & 1.656 \\
\hline & & & 0.85 & 1249 & 394 & 285 & 1.382 & & & & 0.85 & 2018 & 523 & 299 & 1.749 \\
\hline & & & 0.90 & 1296 & 426 & 290 & 1.469 & & & & 0.90 & 2081 & 561 & 304 & 1.845 \\
\hline & & 0.50 & 0.75 & 292 & 85 & 69 & 1.232 & & & 0.50 & 0.75 & 476 & 116 & 72 & 1.611 \\
\hline & & & 0.80 & 303 & 93 & 70 & 1.329 & & & & 0.80 & 491 & 121 & 74 & 1.635 \\
\hline & & & 0.85 & 315 & 99 & 72 & 1.375 & & & & 0.85 & 507 & 132 & 75 & 1.760 \\
\hline & & & 0.90 & 326 & 107 & 73 & 1.466 & & & & 0.90 & 522 & 142 & 76 & 1.868 \\
\hline 4 & 0.70 & 0.25 & 0.75 & 1038 & 250 & 197 & 1.269 & 6 & 0.70 & 0.25 & 0.75 & 1603 & 307 & 216 & 1.189 \\
\hline & & & 0.80 & 1072 & 268 & 201 & 1.333 & & & & 0.80 & 1647 & 333 & 219 & 1.351 \\
\hline & & & 0.85 & 1106 & 290 & 204 & 1.422 & & & & 0.85 & 1692 & 354 & 223 & 1.421 \\
\hline & & & 0.90 & 1141 & 309 & 208 & 1.486 & & & & 0.90 & 1736 & 380 & 226 & 1.488 \\
\hline & & 0.50 & 0.75 & 261 & 65 & 49 & 1.327 & & & 0.50 & 0.75 & 403 & 79 & 54 & 1.224 \\
\hline & & & 0.80 & 269 & 69 & 50 & 1.380 & & & & 0.80 & 414 & 84 & 55 & 1.312 \\
\hline & & & 0.85 & 278 & 74 & 51 & 1.451 & & & & 0.85 & 425 & 89 & 56 & 1.397 \\
\hline & & & 0.90 & 287 & 79 & 52 & 1.519 & & & & 0.90 & 437 & 95 & 57 & 1.466 \\
\hline & 0.80 & 0.25 & 0.75 & 1230 & 306 & 231 & 1.325 & & 0.80 & 0.25 & 0.75 & 1862 & 386 & 246 & 1.280 \\
\hline & & & 0.80 & 1272 & 332 & 235 & 1.413 & & & & 0.80 & 1916 & 416 & 250 & 1.353 \\
\hline & & & 0.85 & 1314 & 358 & 219 & 1.635 & & & & 0.85 & 1970 & 446 & 254 & 1.442 \\
\hline & & & 0.90 & 1357 & 385 & 243 & 1.584 & & & & 0.90 & 2024 & 476 & 258 & 1.473 \\
\hline & & 0.50 & 0.75 & 309 & 77 & 58 & 1.328 & & & 0.50 & 0.75 & 468 & 102 & 61 & 1.273 \\
\hline & & & 0.80 & 320 & 84 & 59 & 1.424 & & & & 0.80 & 481 & 109 & 62 & 1.372 \\
\hline & & & 0.85 & 330 & 90 & 60 & 1.500 & & & & 0.85 & 495 & 111 & 64 & 1.413 \\
\hline & & & 0.90 & 341 & 97 & 61 & 1.590 & & & & 0.90 & 509 & 119 & 65 & 1.514 \\
\hline & 0.90 & 0.25 & 0.75 & 1528 & 396 & 283 & 1.399 & & 0.90 & 0.25 & 0.75 & 2267 & 503 & 294 & 1.370 \\
\hline & & & 0.80 & 1581 & 429 & 288 & 1.490 & & & & 0.80 & 2335 & 541 & 299 & 1.429 \\
\hline & & & 0.85 & 1635 & 463 & 293 & 1.580 & & & & 0.85 & 2404 & 580 & 304 & 1.500 \\
\hline & & & 0.90 & 1690 & 498 & 298 & 1.671 & & & & 0.90 & 2474 & 620 & 309 & 1.618 \\
\hline & & 0.50 & 0.75 & 384 & 100 & 71 & 1.408 & & & 0.50 & 0.75 & 569 & 125 & 74 & 1.355 \\
\hline & & & 0.80 & 397 & 109 & 72 & $1.5+8$ & & & & 0.80 & 586 & 136 & 75 & 1.421 \\
\hline & & & 0.85 & 411 & 119 & 73 & 1.630 & & & & 0.85 & 603 & 247 & 76 & 1.510 \\
\hline & & & 0.90 & 425 & 125 & 75 & 1.667 & & & & 0.90 & 620 & 158 & 77 & 1.605 \\
\hline
\end{tabular}


Table 2: Exact (Discrete) Optimum Sample Sizes for Linear Response $(\delta=0.05, \alpha=0.05)$

\begin{tabular}{|c|c|c|c|c|c|c|c|c|c|c|c|c|c|c|c|}
\hline$k$ & $1-\beta$ & $\eta$ & $\lambda$ & $N$ & $n_{0}$ & $n$ & $r$ & $k$ & $1-\beta$ & $\eta$ & $\lambda$ & $N$ & $n_{0}$ & $n$ & $r$ \\
\hline \multirow[t]{24}{*}{3} & \multirow[t]{8}{*}{0.70} & 0.25 & 0.75 & 465 & 129 & 112 & 1.152 & \multirow[t]{24}{*}{5} & \multirow[t]{8}{*}{0.70} & \multirow[t]{4}{*}{0.25} & 0.75 & 692 & 147 & 109 & 1.349 \\
\hline & & & 0.80 & 489 & 141 & 116 & 1.216 & & & & 0.80 & 733 & 163 & 114 & 1.430 \\
\hline & & & 0.85 & 516 & 156 & 120 & 1.300 & & & & 0.85 & 798 & 188 & 122 & 1.541 \\
\hline & & & 0.90 & 587 & 182 & 135 & 1.348 & & & & 0.90 & 939 & 229 & 142 & 1.613 \\
\hline & & \multirow[t]{4}{*}{0.50} & 0.75 & 118 & 34 & 28 & 1.214 & & & \multirow[t]{4}{*}{0.50} & 0.75 & 173 & 38 & 27 & 1.407 \\
\hline & & & 0.80 & 123 & 36 & 29 & 1.241 & & & & 0.80 & 183 & 43 & 28 & 1.536 \\
\hline & & & 0.85 & 130 & 40 & 30 & 1.333 & & & & 0.85 & 200 & 50 & 30 & 1.667 \\
\hline & & & 0.90 & 146 & 47 & 33 & 1.424 & & & & 0.90 & 235 & 60 & 35 & 1.714 \\
\hline & \multirow[t]{8}{*}{0.80} & 0.25 & 0.75 & 614 & 176 & 146 & 1.205 & & \multirow[t]{8}{*}{0.80} & \multirow[t]{4}{*}{0.25} & 0.75 & 895 & 205 & 138 & 1.486 \\
\hline & & & 0.80 & 639 & 189 & 150 & 1.260 & & & & 0.80 & 936 & 221 & 143 & 1.545 \\
\hline & & & 0.85 & 668 & 206 & 154 & 1.338 & & & & 0.85 & 1000 & 250 & 150 & 1.667 \\
\hline & & & 0.90 & 733 & 235 & 166 & 1.416 & & & & 0.90 & 1141 & 296 & 169 & 1.751 \\
\hline & & \multirow[t]{4}{*}{0.50} & 0.75 & 155 & 44 & 37 & 1.189 & & & \multirow[t]{4}{*}{0.50} & 0.75 & 224 & 54 & 34 & 1.588 \\
\hline & & & 0.80 & 161 & 50 & 37 & 1.351 & & & & 0.80 & 234 & 59 & 35 & 1.686 \\
\hline & & & 0.85 & 168 & 54 & 38 & 1.421 & & & & 0.85 & 252 & 62 & 38 & 1.632 \\
\hline & & & 0.90 & 184 & 61 & 41 & 1.488 & & & & 0.90 & 286 & 76 & 42 & 1.810 \\
\hline & \multirow[t]{8}{*}{0.90} & 0.25 & 0.75 & 849 & 246 & 201 & 1.224 & & \multirow[t]{8}{*}{0.90} & \multirow[t]{4}{*}{0.25} & 0.75 & 1232 & 292 & 188 & 1.553 \\
\hline & & & 0.80 & 884 & 269 & 205 & 1.312 & & & & 0.80 & 1277 & 317 & 192 & 1.651 \\
\hline & & & 0.85 & 919 & 292 & 209 & 1.397 & & & & 0.85 & 1338 & 348 & 198 & 1.758 \\
\hline & & & 0.90 & 978 & 321 & 219 & 1.466 & & & & 0.90 & 1472 & 397 & 215 & 1.847 \\
\hline & & \multirow[t]{4}{*}{0.50} & 0.75 & 214 & 64 & 50 & 1.280 & & & \multirow[t]{4}{*}{0.50} & 0.75 & 310 & 75 & 47 & 1.596 \\
\hline & & & 0.80 & 222 & 69 & 51 & 1.353 & & & & 0.80 & 321 & 81 & 48 & 1.688 \\
\hline & & & 0.85 & 231 & 75 & 52 & 1.442 & & & & 0.85 & 336 & 91 & 49 & 1.857 \\
\hline & & & 0.90 & 246 & 81 & 55 & 1.473 & & & & 0.90 & 370 & 100 & 54 & 1.852 \\
\hline 4 & 0.70 & 0.25 & 0.75 & 580 & 140 & 110 & 1.273 & 6 & 0.70 & 0.25 & 0.75 & 806 & 158 & 108 & 1.429 \\
\hline & & & 0.80 & 607 & 155 & 113 & 1.372 & & & & 0.80 & 860 & 176 & 114 & 1.500 \\
\hline & & & 0.85 & 655 & 171 & 121 & 1.413 & & & & 0.85 & 945 & 201 & 124 & 1.500 \\
\hline & & & 0.90 & 761 & 209 & 138 & 1.514 & & & & 0.90 & 1121 & 245 & 146 & 1.595 \\
\hline & & 0.50 & 0.75 & 145 & 37 & 27 & 1.370 & & & 0.50 & 0.75 & 202 & 40 & 27 & 1.404 \\
\hline & & & 0.80 & 152 & 40 & 28 & 1.429 & & & & 0.80 & 215 & 47 & 28 & 1.487 \\
\hline & & & 0.85 & 165 & 45 & 30 & 1.500 & & & & 0.85 & 237 & 51 & 31 & 1.579 \\
\hline & & & 0.90 & 191 & 55 & 34 & 1.618 & & & & 0.90 & 281 & 65 & 36 & 1.662 \\
\hline & 0.80 & 0.25 & 0.75 & 755 & 191 & 141 & 1.355 & & 0.80 & 0.25 & 0.75 & 1033 & 217 & 136 & 1.207 \\
\hline & & & 0.80 & 786 & 206 & 145 & 1.421 & & & & 0.80 & 1085 & 239 & 141 & 1.551 \\
\hline & & & 0.85 & 832 & 228 & 151 & 1.510 & & & & 0.85 & 1171 & 265 & 151 & 1.569 \\
\hline & & & 0.90 & 936 & 268 & 167 & 1.605 & & & & 0.90 & 1347 & 321 & 171 & 1.704 \\
\hline & & 0.50 & 0.75 & 190 & 50 & 35 & 1.429 & & & 0.50 & 0.75 & 259 & 55 & 34 & 1.349 \\
\hline & & & 0.80 & 198 & 54 & 36 & 1.500 & & & & 0.80 & 272 & 62 & 35 & 1.430 \\
\hline & & & 0.85 & 209 & 57 & 38 & 1.500 & & & & 0.85 & 294 & 72 & 37 & 1.541 \\
\hline & & & 0.90 & 235 & 67 & 42 & 1.595 & & & & 0.90 & 339 & 81 & 43 & 1.613 \\
\hline & 0.90 & 0.25 & 0.75 & 1043 & 271 & 193 & 1.404 & & 0.90 & 0.25 & 0.75 & 1415 & 311 & 184 & 1.407 \\
\hline & & & 0.80 & 1081 & 293 & 197 & 1.487 & & & & 0.80 & 1469 & 341 & 188 & 1.536 \\
\hline & & & 0.85 & 1127 & 319 & 202 & 1.579 & & & & 0.85 & 1549 & 273 & 196 & 1.667 \\
\hline & & & 0.90 & 1223 & 359 & 216 & 1.662 & & & & 0.90 & 1721 & 431 & 215 & 1.714 \\
\hline & & 0.50 & 0.75 & 262 & 70 & 58 & 1.207 & & & 0.50 & 0.75 & 356 & 80 & 46 & 1.486 \\
\hline & & & 0.80 & 272 & 76 & 49 & 1.554 & & & & 0.80 & 369 & 87 & 47 & 1.545 \\
\hline & & & 0.85 & 284 & 80 & 51 & 1.569 & & & & 0.85 & 389 & 95 & 49 & 1.667 \\
\hline & & & 0.90 & 308 & 92 & 54 & 1.704 & & & & 0.90 & 433 & 109 & 54 & 1.751 \\
\hline
\end{tabular}


Table 3: Approximate (Continuous) Optimum Sample Sizes for Step Response Case $(\alpha=$ 0.05 )

\begin{tabular}{|c|c|c|c|c|c|c|c|c|c|}
\hline$k$ & $1-\beta$ & $\lambda$ & $\gamma$ & $r$ & $k$ & $1-\beta$ & $\lambda$ & $\gamma$ & $r$ \\
\hline \multirow[t]{12}{*}{3} & \multirow[t]{4}{*}{0.70} & 0.75 & 6.883 & 1.137 & \multirow[t]{12}{*}{5} & \multirow[t]{4}{*}{0.70} & 0.75 & 9.056 & 1.347 \\
\hline & & 0.80 & 7.009 & 1.210 & & & 0.80 & 9.190 & 1.429 \\
\hline & & 0.85 & 7.135 & 1.282 & & & 0.85 & 9.323 & 1.510 \\
\hline & & 0.90 & 7.260 & 1.354 & & & 0.90 & 9.456 & 1.590 \\
\hline & \multirow[t]{4}{*}{0.80} & 0.75 & 7.556 & 1.182 & & \multirow[t]{4}{*}{0.80} & 0.75 & 9.807 & 1.454 \\
\hline & & 0.80 & 7.698 & 1.258 & & & 0.80 & 9.957 & 1.544 \\
\hline & & 0.85 & 7.840 & 1.334 & & & 0.85 & 10.108 & 1.634 \\
\hline & & 0.90 & 7.982 & 1.409 & & & 0.90 & 10.258 & 1.722 \\
\hline & \multirow[t]{4}{*}{0.90} & 0.75 & 8.498 & 1.228 & & \multirow[t]{4}{*}{0.90} & 0.75 & 10.866 & 1.552 \\
\hline & & 0.80 & 8.662 & 1.307 & & & 0.80 & 11.039 & 1.650 \\
\hline & & 0.85 & 8.826 & 1.386 & & & 0.85 & 11.212 & 1.748 \\
\hline & & 0.90 & 8.990 & 1.465 & & & 0.90 & 11.384 & 1.845 \\
\hline \multirow[t]{12}{*}{4} & \multirow[t]{4}{*}{0.70} & 0.75 & 8.032 & 1.257 & \multirow[t]{12}{*}{6} & \multirow[t]{4}{*}{0.70} & 0.75 & 9.992 & 1.414 \\
\hline & & 0.80 & 8.162 & 1.336 & & & 0.80 & 10.129 & 1.497 \\
\hline & & 0.85 & 8.292 & 1.414 & & & 0.85 & 10.266 & 1.579 \\
\hline & & 0.90 & 8.422 & 1.492 & & & 0.90 & 10.402 & 1.661 \\
\hline & \multirow[t]{4}{*}{0.80} & 0.75 & 8.747 & 1.330 & & \multirow[t]{4}{*}{0.80} & 0.75 & 10.774 & 1.561 \\
\hline & & 0.80 & 8.894 & 1.415 & & & 0.80 & 10.927 & 1.656 \\
\hline & & 0.85 & 9.041 & 1.498 & & & 0.85 & 11.080 & 1.750 \\
\hline & & 0.90 & 9.187 & 1.581 & & & 0.90 & 11.233 & 1.843 \\
\hline & \multirow[t]{4}{*}{0.90} & 0.75 & 9.752 & 1.400 & & \multirow[t]{4}{*}{0.90} & 0.75 & 11.881 & 1.687 \\
\hline & & 0.80 & 9.921 & 1.49 & & & 0.80 & 12.057 & 1.793 \\
\hline & & 0.85 & 10.090 & 1.579 & & & 0.85 & 12.232 & 1.898 \\
\hline & & 0.90 & 10.259 & 1.668 & & & 0.90 & 12.407 & 2.003 \\
\hline
\end{tabular}

